\title{
Stereotactic radiosurgery for spinal metastases with or without separation surgery
}

\author{
Berkeley G. Bate, MD, ${ }^{1}$ Nickalus R. Khan, MD, ${ }^{1}$ Brent Y. Kimball, MD, ${ }^{1}$ Kyle Gabrick, BSc, ${ }^{2}$ \\ and Jason Weaver, MD ${ }^{1,3}$ \\ 1Department of Neurosurgery, University of Tennessee Health Science Center; ${ }^{2}$ College of Medicine, University of Tennessee \\ Health Science Center; and ${ }^{3}$ Semmes-Murphey Neurologic \& Spine Institute, Memphis, Tennessee
}

\begin{abstract}
OBJECT In patients with significant epidural spinal cord compression, initial surgical decompression and stabilization of spinal metastases, as opposed to radical oncological resection, provides a margin around the spinal cord that facilitates subsequent treatment with high-dose adjuvant stereotactic radiosurgery (SRS). If a safe margin exists between tumor and spinal cord on initial imaging, then high-dose SRS may be used as the primary therapy, eliminating the need for surgery. Selecting the appropriate approach has shown greater efficacy of tumor control, neurological outcome, and duration of response when compared with external beam radiotherapy, regardless of tumor histology. This study evaluates the efficacy of this treatment approach in a series of 57 consecutive patients.

METHODS Patients treated for spinal metastases between 2007 and 2011 using the Varian Trilogy Linear Accelerator were identified retrospectively. Each received SRS, with or without initial surgical decompression and instrumentation. Medical records were reviewed to assess neurological outcome and surgical or radiation-induced complications. Magnetic resonance images were obtained for each patient at 3-month intervals posttreatment, and radiographic response was assessed as stability/regression or progression. End points were neurological outcome and local radiographic disease control at death or latest follow-up.
\end{abstract}

RESULTS Fifty-seven patients with 69 lesions were treated with SRS for spinal metastases. Forty-eight cases $(70 \%)$ were treated with SRS alone, and $21(30 \%)$ were treated with surgery prior to SRS. A single fraction was delivered in 38 cases (55\%), while a hypofractionated scheme was used in 31 (45\%). The most common histological entities were renal cell, breast, and lung carcinomas. Radiographically, local disease was unchanged or regressed in 63 of 69 tumors (91.3\%). Frankel score improved or remained stable in 68 of 69 cases (98.6\%).

CONCLUSIONS SRS, alone or as an adjunct following surgical decompression, provides durable local radiographic disease control while preserving or improving neurological function. This less-invasive alternative to radical spinal oncological resection appears to be effective regardless of tumor histology without sacrificing durability of radiographic or clinical response.

http://thejns.org/doi/abs/10.3171/2014.10.SPINE14252

KEY WORDS radiosurgery; spine; metastasis; metastases; decompression; instrumentation; local control; separation surgery; hypofractionated; single fraction; oncology

$\mathrm{M}$ ORE than 1.6 million new cases of cancer are diagnosed annually in the United States. ${ }^{2,24}$ Symptomatic spinal metastases are estimated to occur in up to $10 \%$ of all cancer patients..$^{28}$ The majority occur in the extradural compartment, most frequently presenting within the vertebral bodies. ${ }^{12}$ Most of the metastatic burden $(70 \%)$ is within the thoracic spine, followed by the lumbar spine (20\%), cervical spine, and sacrum..$^{20}$ As survival rates for primary tumors improve, it is expected that the prevalence of spinal metastases will continue to increase. ${ }^{6}$

The treatment paradigm for management of spinal metastatic disease has shifted dramatically over the last 2 decades. ${ }^{5-7}$ Conventional external beam radiation thera-

ABBREVIATIONS cEBRT = conventional external beam radiation therapy; CTV = clinical tumor volume; ESCC = epidural spinal cord compression; GTV = gross tumor volume; $S R S=$ stereotactic radiosurgery.

SUBMITTED March 10, 2014. ACCEPTED October 6, 2014.

INCLUDE WHEN CITING Published online January 30, 2015; DOI: 10.3171/2014.10.SPINE14252.

DISCLOSURE The authors report no conflict of interest concerning the materials or methods used in this study or the findings specified in this paper. 
py (cEBRT) has been the principal method of delivering radiation to the metastatic spine. ${ }^{16}$ However, cEBRT lacks the precision to deliver large radiation doses to the spine without also exposing nearby radiosensitive structures such as the spinal cord. This limits the ability of cEBRT to deliver a cytotoxic dose to a tumor., ${ }^{9,11,14,15,22-25}$

Stereotactic radiosurgery (SRS), on the other hand, delivers high-dose radiation to spinal metastases while limiting irradiation of the spinal cord..$^{10,25,27}$ SRS has been shown through multiple studies to be accurate, safe, and effective in providing local tumor control and alleviating pain. ${ }^{1,8,10,21,23,30}$ SRS therapy has also been shown to improve outcomes independent of tumor histology, which previously had been a limitation with cEBRT as described above. ${ }^{18}$

Surgical treatments have undergone a similar evolution, ${ }^{13}$ and surgical strategy has been influenced by the success of radiotherapy. In 2005, Patchell et al. ${ }^{18}$ demonstrated the superiority of surgical decompression and instrumentation followed by conventional radiation therapy versus radiation therapy alone.

Radiosurgery alone has recently been shown to be an effective noninvasive treatment for spinal metastases with limited epidural compression..$^{10,27}$ In cases of higher-grade epidural compression, however, radiosurgery may not offer a safe or effective strategy for tumor control and decompression of neural elements. For these cases, operative decompression can be used as a tool to allow for appropriate radiosurgical treatment. This method has been shown to be a safe and effective method for local disease control.5,6,8,14

The current paradigm at our institution, as described by Bilsky and colleagues, ${ }^{5-8,14}$ is to consider each patient in terms of neurological function, oncological histology and prognosis, the mechanical stability of the spine, and systemic disease and medical comorbidities (NOMS). A patient's neurological function is usually directly related to the degree of epidural spinal cord compression (ESCC) caused by tumor growth (Fig. 1). Those with high-grade ESCC and/or mechanical instability undergo debulking and instrumentation, or "separation surgery," to create a tumor-free margin around the thecal sac and to stabilize the spine. ${ }^{6,18}$ High-dose SRS may be used as the primary therapy if a safe margin exists between the spinal cord and tumor on initial imaging, eliminating the need for surgery.
This paradigm has shown greater efficacy for tumor control, neurological outcome, and duration of response when compared with cEBRT, regardless of tumor histology. ${ }^{16}$ In this study, we evaluated the efficacy of radiosurgery with or without separation surgery for treatment of oligometastatic disease at our institution in a series of consecutive patients.

\section{Methods \\ Study Design}

An institutional review board-approved retrospective chart review from January 1, 2007, to December 31, 2011, was undertaken to identify patients treated at our institution for metastatic spine disease who had ESCC and underwent either radiosurgery alone or surgical decompression followed by adjuvant radiosurgery. In instances in which patients were treated for more than 1 lesion, each focus of treatment was considered as a separate case in our analysis.

\section{Surgery}

Patients with high-grade ESCC (Grades 2 and 3, Fig. 1) or those with a pathological fracture and clinical signs of mechanical instability underwent separation surgery. A single surgeon (J.W.) treated all lesions. The primary goal of surgery was epidural decompression and spinal stabilization. Gross-total resection was not attempted in any case. Surgery consisted of epidural tumor debulking and partial or complete vertebrectomy from a posterolateral approach. Pedicle and/or lateral mass screw fixation as well as placement of titanium cages or methyl methacrylate were used as appropriate. Instrumentation was placed at least 2 levels above and below the level of decompression. Titanium anterior support implants were used in a minority of cases. Local autologous bone and allograft were used in all patients.

\section{Stereotactic Radiosurgery}

Patients presenting with low-grade ESCC (Grades 0 and 1, Fig. 1) without evidence of mechanical instability underwent SRS as primary therapy using the Varian Trilogy Linear Accelerator (Varian Medical Systems, Inc.). For these patients, MRI was blended to CT simulations for

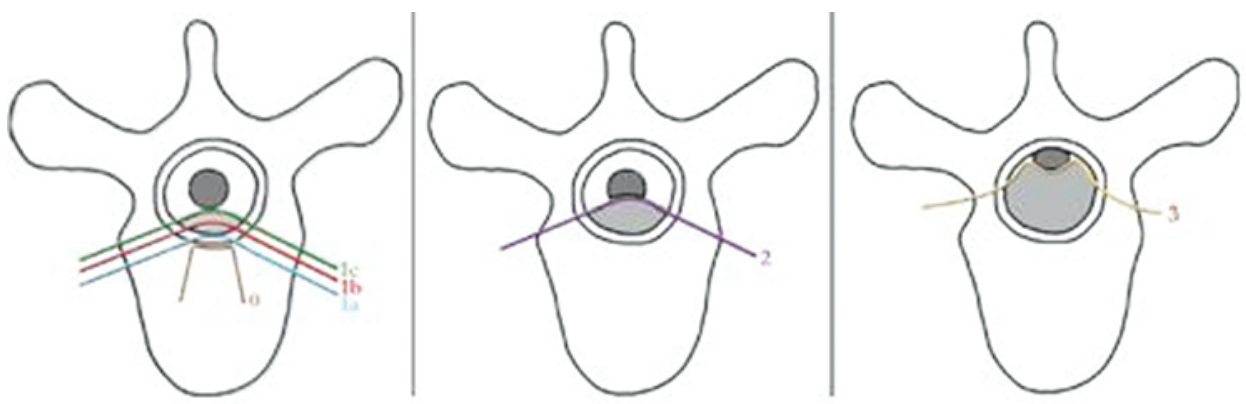

FIG. 1. Grading of ESCC. Left: Grade 0 is disease confined to bone, Grade 1a disease involves the epidural space but does not compress the dura, Grade $1 \mathrm{~b}$ disease compresses the dura but does not abut the spinal cord, and Grade $1 \mathrm{c}$ disease abuts but does not compress or adjust the course of the spinal cord. Center: Grade 2 disease compresses the spinal cord, but CSF is still visible. Right: Grade 3 disease compresses the spinal cord, obliterating all visible CSF at that level. (Reproduced with permission from Moulding et al: $J$ Neurosurg Spine 13:87-93, 2010). Figure is available in color online only. 
planning purposes. Gross tumor volume (GTV) was contoured to include all osseous, paraspinous, and epidural tumors. Clinical tumor volume (CTV) was contoured to include any contiguous nondiseased elements of the vertebral body. In general, the CTV prescription dose was $80 \%$ of that delivered to the GTV.

For patients with high-grade ESCC, CT myelography performed postoperatively was used to contour a GTV that included any residual epidural and paraspinal disease. A CTV was contoured to preoperative tumor volume and contiguous elements of the diseased vertebral body rather than residual disease, as described by Bilsky. ${ }^{5}$

Image-guided radiotherapy was given as a single fraction or in a hypofractionated scheme. Because the radiosurgery literature has demonstrated consistent efficacy regardless of tumor histology, dose prescription was made independent of histology. The dose and fractionation scheme was based on tumor volume and extent of epidural disease. Epidural disease limited to Grade 0 or 1 spanning fewer than 3 vertebral segments was typically treated with a single fraction, ranging from 16 to $23 \mathrm{~Gy}$. The initial dosing in our series was 16 Gy in a single fraction. To obtain increased efficacy, dosing was escalated after safety was established. The decision to hypofractionate the dose was based on the extent of disease or a history of previous radiotherapy at the same segment. In general, if the volume of tumor involved 3 or more vertebral segments, a 3-fraction scheme was used. If radiotherapy had previously been performed, a 5-fraction scheme was used. Patients in whom surgery had been performed were generally treated in a single fraction unless the above conditions warranted fractionation.

Prior to 2010, the maximum point dose to the spinal cord was 12 Gy for single fractions, 14 Gy for 3 fractions, and 9 Gy for 5 fractions. In 2010, a series of guidelines published by the American Association of Physicists in Medicine Task Group $101^{3}$ influenced our treatment paradigm. The maximum radiation point dose to the spinal cord after the implementation of these guidelines was 14 Gy for single-fraction treatments, 21.9 Gy for 3 fractions, and 30 Gy for 5 fractions.

\section{Imaging and Follow-Up}

Patients underwent follow-up at 3-month intervals after SRS with serial gadolinium-enhanced MRI. These images were reviewed at the time of treatment by the senior surgeon (J.W.) and a neuroradiologist, and based on the findings tumors were assigned to one of 2 categories: 1) regression or stability of local tumor volume or 2) local progression of disease. The date of last imaging or the date of imaging showing local recurrence was used to determine the duration of local control. Date of death or last clinic visit was used to determine the length of follow-up. End points were neurological outcome and local radiographic disease control.

\section{Statistical Analysis}

The Kaplan-Meier method was used to create estimates of overall survival. Patients were censored according to follow-up and death in calculations of percentile risk.
Confidence intervals were calculated using an alpha value of 0.05 . A univariate regression analysis was performed to assess the significance of factors that might influence the primary end points. Statistics were generated using SPSS (version 21, IBM).

\section{Results}

A total of 63 patients treated from January 1, 2007, through December 31, 2011, were identified, but 6 of these patients were lost to follow-up. Therefore, a total of 57 patients with 69 lesions were included in this analysis. Twenty-one lesions were treated with surgical decompression followed by SRS, and 48 lesions were treated with SRS alone. At patient death or latest follow-up, 63 of 69 cases $(91.3 \%)$ had local disease control, and the remaining 6 cases $(8.7 \%)$ demonstrated radiographic progression. Tables 1 and 2 show patient demographics and locations of the tumors treated. The majority of cases $(65 \%)$ involved single-level disease and 22\% had 2-level disease. Table 3 shows the histology and rates of local failure of the tumors treated. The 6 patients with local recurrence are described in Table 4; 3 were from the SRS+surgery group and 3 were from the SRS-only group. The mean time to recurrence was 8.1 months (range 2.5-16.6 months). There were recurrences within each of the fractionation schemes in the study. Three instances were found on surveillance imaging and were asymptomatic, and one of these actually occurred outside the SRS field in the paraspinous musculature. Two patients presented with radicular symptoms and worsening pain. One patient had imaging studies as part of a workup for a new foot drop, which revealed recurrence, but the foot drop was ultimately found to be caused by peroneal nerve compression in the popliteal fossa. Further treatment was determined on a case-by-case basis and consisted of observation, resection, or re-resection as needed.

A univariate regression analysis was performed to assess which factors might predict recurrence. The following variables were analyzed: treatment type (SRS alone vs SRS+surgery), age ( $<60$ vs $\geq 60$ years), histology, region of spine involvement, number of levels treated, prior conventional radiation, fractionation (hypofractionated vs single), and dose (hypofractionated 20,27, or 30 Gy and single fraction 16 Gy vs $20-22$ Gy). None of the variables were found to be statistically significant factors in predicting recurrence.

The median length of follow-up was 9.8 months in the SRS-only group, 13.7 months in the SRS+surgery group, and 10 months overall. Of the 48 cases in the SRS-only group, $43(90 \%)$ had a stable Frankel grade, and the re-

TABLE 1. Patient demographics

\begin{tabular}{lccc}
\hline \multicolumn{1}{c}{ Variable } & SRS+Surgery & SRS & Total \\
\hline Mean age in yrs (range) & $60(35-79)$ & $59.8(29-81)$ & $59.8(29-81)$ \\
\hline No. of lesions & 21 & 48 & 69 \\
\hline No. in males (\%) & $10(33)$ & $20(66)$ & 30 \\
\hline No. in females (\%) & $11(28)$ & $28(72)$ & 39 \\
\hline No. w/ prior cEBRT (\%) & $6(20)$ & $24(80)$ & 30 \\
\hline
\end{tabular}


TABLE 2. Tumor location and local failure

\begin{tabular}{lcc}
\hline \multicolumn{1}{c}{ Location } & Total & Local Failure $(\%)$ \\
\hline Cervical & 9 & 0 \\
\hline Cervicothoracic & 2 & $1(50)$ \\
\hline Thoracic & 34 & $2(6)$ \\
\hline Thoracolumbar & 3 & 0 \\
\hline Lumbar & 19 & $3(16)$ \\
\hline Sacral & 2 & 0 \\
\hline
\end{tabular}

maining 10\% had an improved Frankel grade. Of the 21 cases in the SRS+surgery group, $17(81 \%)$ had a stable Frankel grade, and 3 (14\%) showed an improved grade. The remaining patient, who had a decline in Frankel grade, developed new cervical radiculopathy that was not due to a compressive lesion.

There were 5 fractures (7.2\%) overall; hypofractionated therapy was performed in 4 of the 5 cases, and single-fraction therapy was performed in the remaining case. None of the patients who received SRS and surgery sustained a pathological fracture. The most common histological findings were renal cell, breast, and lung carcinomas, which accounted for $66 \%$ of the cases (Table 3). There was 1 recurrence in the breast/lung/renal cell group. There was 1 case of mild esophagitis that resolved within 3 months. There were no cases of radiation-induced myelopathy. There were 2 cases of durotomy in the surgical group, both of which were closed primarily with no further sequelae. Data on pain were available for 45 of 69 cases $(65 \%)$, with an overall average visual analog scale score decrease of $3.4 \pm 2.6$ (median 3 ) following treatment.

The median overall time to recurrence was 9.6 months (range 2.5-16.6 months). A total of 32 patients (56\%, median follow-up 6.6 months) died; of these, 3 had local

TABLE 3. Tumor histology and local failure

\begin{tabular}{lcc}
\hline \multicolumn{1}{c}{ Carcinoma } & Total & Local Failure (\%) \\
\hline Renal cell & 18 & $1(6)$ \\
\hline Breast & 17 & 0 \\
\hline Lung & 11 & 0 \\
\hline Bladder & 1 & 0 \\
\hline Colon & 1 & 0 \\
\hline Hepatocellular & 1 & 0 \\
\hline Melanoma & 2 & $1(50)$ \\
\hline Neuroendocrine & 1 & 0 \\
\hline Ovarian & 1 & $1(100)$ \\
\hline Pancreatic & 2 & $1(50)$ \\
\hline Parotid & 1 & $1(100)$ \\
\hline Prostate & 4 & 0 \\
\hline Sarcoma & 3 & 0 \\
\hline Squamous cell head/neck & 2 & 0 \\
\hline Thyroid & 1 & 0 \\
\hline Uterine & 2 & 0 \\
\hline Vulvovaginal & 1 & $1(100)$ \\
\hline
\end{tabular}


progression. Twenty-five patients (44\%) were alive with a median follow-up of 15.4 months. Of the patients still living, $3(12 \%)$ had local progression of their disease. The remaining 22 patients were alive and free of local failure at a median follow-up of 15.6 months. A Kaplan-Meier survival analysis was performed to demonstrate the time to local failure (Fig. 2).

Overall, there was a 5.8\% risk of local failure at 1 year. Tables 5 and 6 show the percentage risk of local failure at 1 year for the SRS-only and SRS+surgery groups and subdivide them by fractionation scheme. The 1-year risk of failure for SRS alone was $4.2 \%$ and for SRS+surgery was $9.5 \%$.

\section{Discussion}

The goals of treatment for metastatic spinal disease are palliative in nature and consist of alleviating pain, providing mechanical stability, maintaining or improving neurological function, and achieving durable local tumor control. Patchell et al. ${ }^{18}$ and Bilsky ${ }^{5}$ demonstrated that surgery combined with cEBRT was superior to radiation therapy alone in achieving these goals. More recently, high-dose, single-fraction SRS has also been shown to be a safe and effective palliative treatment independent of tumor histology. ${ }^{1,10,22,27,29}$ SRS following separation surgery is also safe and effective. ${ }^{17,19}$ A study in 2010 demonstrated better results for those receiving higher doses of SRS following surgery. ${ }^{17}$

Recently, Laufer et al..$^{14}$ reported a series of 186 patients with ESCC treated with SRS and surgery. This is the largest series to date showing the efficacy of separation surgery followed by adjuvant SRS. Importantly, the authors demonstrated that high-dose hypofractionated (24-30 Gy in 3 fractions) therapy provided a benefit in local tumor control compared with low-dose (18-36 Gy in 5 to 6 fractions) hypofractionated adjuvant therapy. The rate of recurrence in the high-dose hypofractionated group was

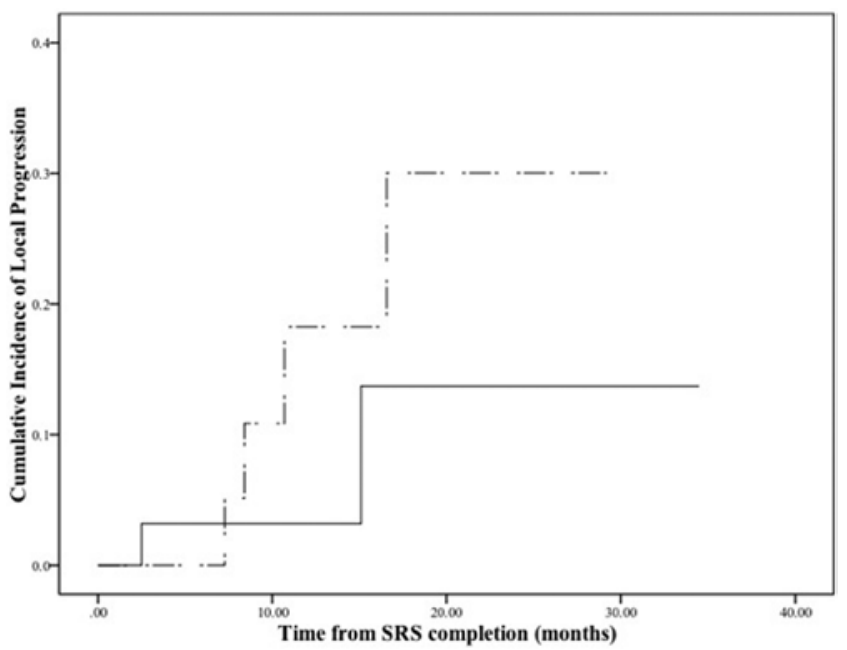

FIG. 2. Cumulative incidence of local progression as a function of time from SRS completion (Kaplan-Meier method). The dashed line represents the group receiving hypofractionated therapy and the solid line represents the group receiving single-fraction SRS.
TABLE 5. One-year percentage risk of local failure: SRS alone

\begin{tabular}{|c|c|c|c|c|}
\hline Group & $\begin{array}{c}\text { No. of } \\
\text { Fractions }\end{array}$ & $\begin{array}{l}\text { No. of } \\
\text { Lesions }\end{array}$ & $\begin{array}{l}\text { Local } \\
\text { Failure } \\
\text { (no.) }\end{array}$ & $\begin{array}{c}\text { 1-Yr \% Risk } \\
\text { of Local } \\
\text { Failure }\end{array}$ \\
\hline All Patients & & 69 & 4 & 5.8 \\
\hline SRS Alone & & 48 & 2 & 4.2 \\
\hline \multicolumn{5}{|c|}{ Single fraction (Gy) } \\
\hline 23 & & 1 & 0 & - \\
\hline 22 & & 17 & 1 & 5.9 \\
\hline 20 & & 1 & 0 & - \\
\hline 16 & & 5 & 0 & - \\
\hline Overall & & 24 & 1 & 4.2 \\
\hline \multicolumn{5}{|c|}{ Hypofractionated (Gy) } \\
\hline 10 & 2 & 3 & 0 & - \\
\hline 9 & 3 & 8 & 1 & 12.5 \\
\hline 6 & 5 & 13 & 0 & - \\
\hline Overall & & 24 & 1 & 4.2 \\
\hline
\end{tabular}

lower than that in the single-fraction group, although it was not statistically significant.

The objective of this study was to evaluate the efficacy of our approach to treatment of oligometastatic disease to the spine. This includes SRS alone for patients with minimal ESCC and separation surgery followed by SRS for patients with high-grade ESCC. Those receiving SRS alone had a 1-year risk of local failure of $4.2 \%$ (95\% CI $1.6 \%-10.1 \%)$. This result is similar to those found in previous studies by Gerstzen et al. ${ }^{10}$ and Yamada et al. ${ }^{29}$

Gerstzen et al. presented 500 consecutive lesions treated with moderate- to high-dose (15-22.5 Gy) singlefraction SRS with a median follow-up of 21 months..$^{10}$ The authors reported long-term tumor control of $88 \%$ for all cases, and $100 \%$ in breast, lung, and renal cell metastases when SRS was delivered as the primary therapy. Our series demonstrates a $95.8 \%$ (46 of 48) local control rate when using SRS alone. Furthermore, after subdividing our

TABLE 6. One-year percentage risk of local failure: SRS+surgery

\begin{tabular}{|c|c|c|c|c|}
\hline Group & $\begin{array}{c}\text { No. of } \\
\text { Fractions }\end{array}$ & $\begin{array}{l}\text { No. of } \\
\text { Lesions }\end{array}$ & $\begin{array}{c}\text { Local } \\
\text { Failure } \\
\text { (no.) }\end{array}$ & $\begin{array}{c}\text { 1-Yr \% Risk } \\
\text { of Local } \\
\text { Failure }\end{array}$ \\
\hline All Patients & & 69 & 4 & 5.8 \\
\hline SRS+surgery & & 21 & 2 & 9.5 \\
\hline \multicolumn{5}{|c|}{ Single fraction (Gy) } \\
\hline 22 & & 9 & 0 & - \\
\hline 20 & & 2 & 0 & - \\
\hline 16 & & 3 & 0 & - \\
\hline Overall & & 14 & 0 & 0 \\
\hline \multicolumn{5}{|c|}{ Hypofractionated (Gy) } \\
\hline 10 & 2 & 1 & 1 & 100 \\
\hline 9 & 3 & 3 & 0 & - \\
\hline 6 & 5 & 3 & 1 & 33.3 \\
\hline Overall & & 7 & 2 & 28.6 \\
\hline
\end{tabular}


patients with breast, lung, and renal cell cancer there was only a single recurrence in a patient with renal cell carcinoma (Table 4). The rates of recurrence in this subgroup are consistent with the findings presented by Gerstzen et al. ${ }^{10}$

In our series there were 21 cases of surgery followed by adjuvant SRS with a 1-year rate of local failure of $9.5 \%$ (Table 6). This is the same rate of failure at 1 year as reported by Moulding et al. in 2010. ${ }^{17}$ Recently, Laufer et al. ${ }^{14}$ reported a series of 186 patients with ESCC treated with SRS and surgery, which is the largest series to date showing the efficacy of separation surgery followed by adjuvant SRS. Importantly, the authors demonstrated that highdose (24-30 Gy in 3 fractions) hypofractionated therapy provided a benefit in local tumor control compared with low-dose (18-36 Gy in 5-6 fractions) hypofractionated adjuvant therapy. Additionally, the rate of recurrence in the high-dose hypofractionated group was lower than the single-fraction group, although not statistically significantly.

Our study shows a difference in local progression between single-fraction and hypofractionated therapy in patients who also underwent surgery (0\% vs $28.6 \%$ 1-year risk of local failure); however, this difference was not statistically significant. Any conclusion about the greater efficacy of single-fraction over hypofractionated radiotherapy may, therefore, be premature based on our results and those from the recent study by Laufer et al. ${ }^{14}$ Additionally, we did not identify any factors that could predict which patients might experience a recurrence.

The limitations of this study include the retrospective nature of data collection, small sample size, and single institution analysis, thus limiting generalizability. Another clear limitation of this study is the limited duration of follow-up. This study population has a limited lifespan because of the nature of metastatic disease. Nonetheless, this should not detract from the demonstrated efficacy of such a palliative approach until the time of death. Further investigations can use these data to develop studies that are more adequately powered to investigate SRS with or without surgery as an effective treatment in preventing local progression and preserving neurological function.

\section{Conclusions}

In this retrospective case series, SRS represents a safe, viable, and effective method in managing spinal metastases independent of tumor histology. It was especially effective for the most common histological entities of breast, lung, and renal cell carcinoma. We identified no predictors of treatment failure in this series. SRS alone was a reliable and effective option in treating patients with low-grade ESCC. In patients with high-grade ESCC requiring surgical decompression, SRS was an effective adjuvant therapy in preventing local progression and preserving or improving neurological function.

\section{References}

1. Ahmed KA, Stauder MC, Miller RC, Bauer HJ, Rose PS, Olivier KR, et al: Stereotactic body radiation therapy in spinal metastases. Int J Radiat Oncol Biol Phys 82:e803-e809, 2012
2. American Cancer Society: Cancer Facts \& Figures 2012. (http://www.cancer.org/research/cancerfactsstatistics/cancer factsfigures2012/) [Accessed December 2, 2014]

3. Benedict SH, Yenice KM, Followill D, Galvin JM, Hinson W, Kavanagh B, et al: Stereotactic body radiation therapy: the report of AAPM Task Group 101. Med Phys 37:4078-4101, 2010 (Erratum in Med Phys 39:563, 2012)

4. Bilsky M, Smith M: Surgical approach to epidural spinal cord compression. Hematol Oncol Clin North Am 20:13071317, 2006

5. Bilsky MH: New therapeutics in spine metastases. Expert Rev Neurother 5:831-840, 2005

6. Bilsky MH, Laufer I, Burch S: Shifting paradigms in the treatment of metastatic spine disease. Spine (Phila Pa 1976) 34 (22 Suppl):S101-S107, 2009

7. Bilsky MH, Lis E, Raizer J, Lee H, Boland P: The diagnosis and treatment of metastatic spinal tumor. Oncologist 4:459469, 1999

8. Bilsky MH, Yamada Y, Yenice KM, Lovelock M, Hunt M, Gutin PH, et al: Intensity-modulated stereotactic radiotherapy of paraspinal tumors: a preliminary report. Neurosurgery 54:823-831, 2004

9. Faul CM, Flickinger JC: The use of radiation in the management of spinal metastases. J Neurooncol 23:149-161, 1995

10. Gerszten PC, Burton SA, Ozhasoglu C, Welch WC: Radiosurgery for spinal metastases: clinical experience in 500 cases from a single institution. Spine (Phila Pa 1976) 32:193-199, 2007

11. Gerszten PC, Burton SA, Quinn AE, Agarwala SS, Kirkwood JM: Radiosurgery for the treatment of spinal melanoma metastases. Stereotact Funct Neurosurg 83:213-221, 2005

12. Khanna AJ, Shindle MK, Wasserman BA, Gokaslan ZL, Gonzales RA, Buchowski JM, et al: Use of magnetic resonance imaging in differentiating compartmental location of spinal tumors. Am J Orthop 34:472-476, 2005

13. Klekamp J, Samii H: Surgical results for spinal metastases. Acta Neurochir (Wien) 140:957-967, 1998

14. Laufer I, Iorgulescu JB, Chapman T, Lis E, Shi W, Zhang Z, et al: Local disease control for spinal metastases following "separation surgery" and adjuvant hypofractionated or highdose single-fraction stereotactic radiosurgery: outcome analysis in 186 patients. J Neurosurg Spine 18:207-214, 2013

15. Loblaw DA, Laperriere NJ: Emergency treatment of malignant extradural spinal cord compression: an evidence-based guideline. J Clin Oncol 16:1613-1624, 1998

16. Maranzano E, Latini P: Effectiveness of radiation therapy without surgery in metastatic spinal cord compression: final results from a prospective trial. Int J Radiat Oncol Biol Phys 32:959-967, 1995

17. Moulding HD, Elder JB, Lis E, Lovelock DM, Zhang Z, Yamada Y, et al: Local disease control after decompressive surgery and adjuvant high-dose single-fraction radiosurgery for spine metastases. J Neurosurg Spine 13:87-93, 2010

18. Patchell RA, Tibbs PA, Regine WF, Payne R, Saris S, Kryscio RJ, et al: Direct decompressive surgical resection in the treatment of spinal cord compression caused by metastatic cancer: a randomised trial. Lancet 366:643-648, 2005

19. Rock JP, Ryu S, Shukairy MS, Yin FF, Sharif A, Schreiber F, et al: Postoperative radiosurgery for malignant spinal tumors. Neurosurgery 58:891-898, 2006

20. Ross J, Brant-Zawadzki M, Moore KR, Crim J, Chen MZ, Katzman GL: Neoplasms, cysts, and other masses, in Ross JS (ed): Diagnostic Imaging: Spine. Manitoba, Canada: Amirsys, 2005, Vol 4, pp 1-126

21. Ryu S, Jin R, Jin JY, Chen Q, Rock J, Anderson J, et al: Pain control by image-guided radiosurgery for solitary spinal metastasis. J Pain Symptom Manage 35:292-298, 2008

22. Ryu S, Rock J, Jain R, Lu M, Anderson J, Jin JY, et al: Radio- 
surgical decompression of metastatic epidural compression. Cancer 116:2250-2257, 2010

23. Ryu SI, Chang SD, Kim DH, Murphy MJ, Le QT, Martin DP, et al: Image-guided hypo-fractionated stereotactic radiosurgery to spinal lesions. Neurosurgery 49:838-846, 2001

24. Sciubba DM, Gokaslan ZL: Diagnosis and management of metastatic spine disease. Surg Oncol 15:141-151, 2006

25. Sciubba DM, Petteys RJ, Dekutoski MB, Fisher CG, Fehlings MG, Ondra SL, et al: Diagnosis and management of metastatic spine disease. A review. J Neurosurg Spine 13:94108,2010

26. Shin DA, Huh R, Chung SS, Rock J, Ryu S: Stereotactic spine radiosurgery for intradural and intramedullary metastasis. Neurosurg Focus 27(6):E10, 2009

27. Sohn S, Chung CK: The role of stereotactic radiosurgery in metastasis to the spine. J Korean Neurosurg Soc 51:1-7, 2012

28. Sundaresan N, Digiacinto GV, Hughes JE, Cafferty M, Vallejo A: Treatment of neoplastic spinal cord compression: results of a prospective study. Neurosurgery 29:645-650, 1991

29. Yamada Y, Bilsky MH, Lovelock DM, Venkatraman ES, Toner S, Johnson J, et al: High-dose, single-fraction imageguided intensity-modulated radiotherapy for metastatic spinal lesions. Int J Radiat Oncol Biol Phys 71:484-490, 2008

30. Yamada Y, Lovelock DM, Yenice KM, Bilsky MH, Hunt MA, Zatcky J, et al: Multifractionated image-guided and stereotactic intensity-modulated radiotherapy of paraspinal tumors: a preliminary report. Int J Radiat Oncol Biol Phys 62:53-61, 2005

\section{Author Contributions}

Conception and design: Weaver, Bate, Khan, Kimball. Acquisition of data: Weaver, Bate, Kimball, Gabrick. Analysis and interpretation of data: Weaver, Bate, Khan, Kimball. Drafting the article: Bate, Khan. Critically revising the article: all authors. Reviewed submitted version of manuscript: all authors. Approved the final version of the manuscript on behalf of all authors: Weaver. Statistical analysis: Khan. Administrative/technical/ material support: Gabrick. Study supervision: Weaver.

\section{Supplemental Information}

\section{Previous Presentations}

Portions of this work were presented as oral presentations at the 2012 CNS Annual Meeting in Chicago, IL, October 6-10, 2012, and at the annual meeting of the Radiosurgery Society, Carlsbad, CA, February 21-23, 2013.

\section{Correspondence}

Jason Weaver, Semmes-Murphey Neurologic \& Spine Institute, 6325 Humphreys Blvd., Memphis, TN 38120. email: jweaver@ semmes-murphey.com. 